\title{
Agarwood formation in Aquilaria beccariana and Aquilaria microcarpa in response to inoculation of newly isolated fungi from Brunei Darussalam
}

\author{
YUMNI HAZIQAH MOHAMMAD, POOJA SHIVANAND, FAIZAH METALI, HUSSEIN TAHA, \\ NUR BAZILAH AFIFAH MATUSSIN, ABDUL MUIZZ AL-AZIM ABDUL-HALIM, \\ ABDUL ZUL'ADLY MOHAIMIN \\ Environmental and Life Sciences Program, Faculty of Science, Universiti Brunei Darussalam. Jln. Tungku Link BE1410, Brunei Darussalam. \\ vemail: pooja.shivanand@ubd.edu.bn
}

Manuscript received: 26 April 2021. Revision accepted: 4 September 2021.

\begin{abstract}
Mohammad YH, Shivanand P, Metali F, Taha H, Matussin NBA, Abdul-Halim AMA, Mohaimin AZ. 2021. Agarwood formation in Aquilaria beccariana and Aquilaria microcarpa in response to inoculation of newly isolated fungi from Brunei Darussalam. Biodiversitas 22: 4131-4138. Aquilaria is an important agarwood-producing tree, but naturally occurring agarwood is rare. Artificial induction method is the most commonly used to produce agarwood in a short time. Out of the 21 species of Aquilaria, Brunei Darussalam houses two species that produce high quality agarwood, namely Aquilaria beccariana and A. microcarpa. The aims of this study are to artificially induce agarwood formation using eight different induction treatments: six fungal inoculant treatments with two control treatments, and to isolate and identify fungal strains from naturally infected agarwood tree using DNA barcoding. Agarwood formation was observed at four different time intervals i.e. 1, 3, 6 and 9 months of post-inoculation. Results exhibited that the area and the total length of discoloration zone were significantly greater at 6 and 9 months of incubation period. Induction treatment did not significantly affect the area and the total length of discoloration. FTIR analysis revealed that two aromatic compounds $(\mathrm{C}-\mathrm{H}$ and $\mathrm{C}-\mathrm{C}$ stretching) were detected at the wavelength ranges of $3000-2800 \mathrm{~cm}^{-1}$, and $1500-1400 \mathrm{~cm}^{-1}$ respectively, and $\mathrm{OH}$ group was detected at $3600-3400 \mathrm{~cm}^{-1}$. Nine fungal strains from Aspergillus, Fusarium, Mucor and Trichosporon were isolated and identified from a naturally infected agarwood, and the last two fungi (Mucor and Trichosporon) were never been previously reported.
\end{abstract}

Keywords: Agarwood formation, Aquilaria, FTIR analysis, fungal diversity, fungal inoculants, incubation period

\section{INTRODUCTION}

Agarwood is a fragrant dark resinous wood, derived from the heartwood of wounded trees of several species of Aquilaria and Gyrinops species belonging to Thymeleaceae (Faizal et al. 2020). Synonymously, it is known by many names, such as chenxiang, eaglewood, gaharu or jinkoh, and is notable worldwide because of its high-priced resin (Cui et al. 2013). The genus Aquilaria is endemic to the Indomalesia region and it is currently protected under the Convention on International Trade in Endangered Species (CITES) and the International Union for Conservation of Nature (IUCN) (Lee and Mohamed 2016).

From the natural phenomenon to the event of technology associated with agarwood formation, many countries around the world show endless efforts in unveiling the underlying mechanisms for developing the best quality of agarwood due to its immense value. Agarwood is famous for its essential oils, expensive handicrafts products, cosmetics products and most important for its medicinal values (Naziz et al. 2019). Without the wound, agarwood is of little value. A wound can be defined as injury to trees from natural causes and man-made. Wounds are caused by many natural activities, such as insect infestations, lightning, and disease infections caused by bacteria and fungi (Xu et al. 2013; Zhang et al. 2014). Injuries to trees may also be man-made such as making a deliberate scratch or holes to tree stems and any other types of intentional physical wounding to trees. With present technology, humans can induce infection in a relatively shorter time frame than natural infection using culturable microorganisms such as bacteria and fungi in laboratories. Such microorganisms play a crucial role in the development of agarwood. Several studies have reported that microbial inoculants induced agarwood formation in various Aquilaria species. Tian et al. (2013) previously inoculated uninfected $A$. sinensis with several inoculants of fungal species, namely Phomopsis, Botryosphaeria, Cylindrocladium and Colletotrichum. While Faizal et al. (2017) inoculated A. malaccensis with Fusarium solani. This study explores the potential of agarwood development using two Aquilaria species (A. beccariana and $A$. microcarpa) by employing artificial induction of microbial inoculants in Brunei Darussalam. According to the IUCN Red List, A. beccariana and A. microcarpa are categorized as vulnerable and endangered, respectively (IUCN Red List of Threatened Species 2020). Therefore, the aims of present study are to induce and assess artificial agarwood formation in two Aquilaria species using eight different induction treatments consisting of six fungal strains and two control treatments, and also to investigate the fungal diversity in a naturally infected $A$. beccariana and $A$. microcarpa. 


\section{MATERIALS AND METHODS}

\section{Aquilaria species and study site}

The study was conducted in the primary mixed dipterocarp forest in Benutan Forest Reserve, Tutong District, Brunei Darussalam (4³8'03.7"N 11444'29.1"E). A total of 12 healthy, uninfected medium-sized (diameter at breast height or DBH of $4-6 \mathrm{~cm}$ ) Aquilaria trees were acquired for this research, with 4 trees of $A$. beccariana and 8 trees A. microcarpa. The taxonomic identification of these species was confirmed by the botanists from Brunei National Herbarium (BRUN), Forestry Department, Ministry of Primary Resources and Tourism (MPRT). However, due to the limited supply and imbalance number of trees per species, the induction treatments were randomly applied to all tree species. A t-test was carried out using data collected from both Aquilaria species after 1 month incubation period and it showed that there was no significant difference in discoloration zone between these species.

\section{Induction treatments}

A total of eight induction treatments i.e. six fungalinoculant treatments and two control treatments were applied per Aquilaria tree. Fungal strains used to produce inoculants in this study were isolated from various habitats in Brunei and previously identified using DNA barcoding (Taha et al. 2020) as UBDFH25 Trichoderma sp. (GenBank accession no. MK116452), UBDFH27 Exophiala sp. (MK116454), UBDFH16 Cladosporium sp. (MK116447), UBDFC07 Aspergillus sp. (MK116442), UBDFM02 Trichoderma sp. (MK116429), and UBDFM06 Penicillium sp. (MK116432). All fungal strains were cultured individually on potato dextrose agar media (PDA; Merck, Germany) and incubated for $2-3$ days at $25 \pm 1$ ${ }^{\circ} \mathrm{C}$. A $6 \mathrm{~mm}$ cork-borer was used to plug out the agar from fully-grown PDA plates of each fungal strain and suspended in $250 \mathrm{ml}$ of sterilized Saboroud Dextrose Broth (SDB; Merck, Germany). The SDB was supplemented with Chloramphenicol and then sterilized at $121{ }^{\circ} \mathrm{C}$ for 20 minutes. The suspensions of fungal strains were incubated at $25{ }^{\circ} \mathrm{C}$ for 5 days with constant shaking conditions. Freshly prepared inoculants were kept aseptically into sterile high-density polyethylene (HDPE) bottles and then placed these bottles in a cool Styrofoam box before inoculation at the field site. The two control treatments consisted of SDB media and no inoculants applied.

\section{Inoculation to Aquilaria trees}

Inoculation technique was adopted from Han and Win (2016) with a slight modification. Before inoculation, each tree was initially marked by strapping a duct tape around its bark starting from the first level of the lower section of the tree, $20 \mathrm{~cm}$ above the ground. There were 8 levels made, up along the middle section of the tree trunk with a $20 \mathrm{~cm}$ gap between each level (Figure 1). Each level represents one induction treatment and four holes were made at each level. Holes of $10 \mathrm{~mm}$ depth were made along the tree trunk at approximate distance of $10 \mathrm{~cm}$ from each other. The prepared inoculants were directly injected into the open holes using $3 \mathrm{ml}$ sterile syringes. A sterile bamboo stopper was plugged into the holes to prevent the invasion of environmental microbes (Zhang et al. 2014) and the trees were incubated for the determined period. Eight induction treatments were applied within a tree and shuffled randomly in all 12 trees (Figure 1). Aquilaria trees were incubated at four different time intervals i.e. 1 month, 3 months, 6 months and 9 months. Each of the eight treatments was performed in triplicates per incubation period, resulting in 3 trees per time interval or incubation period.

\section{Measurement of discoloration zone}

Agarwood formation was confirmed by dark discoloration on the infected area or the inoculated site, which was easily observed after peeling the bark off using assorted chisels. The length of discoloration was measured according to the method of Nobuchi and Siripatanadilok (2008), where the measurements were taken from both the upper vertical and lower vertical darker areas. Further, photos of the infected area around the holes were also taken for all induction treatments. Each photo was uploaded into software ImageJ2 (Rueden et al. 2017) to measure the area of discoloration zone. After measurement, agarwood formed on the tree barks was harvested using assorted chisels and stored in a sterile zip-bag.

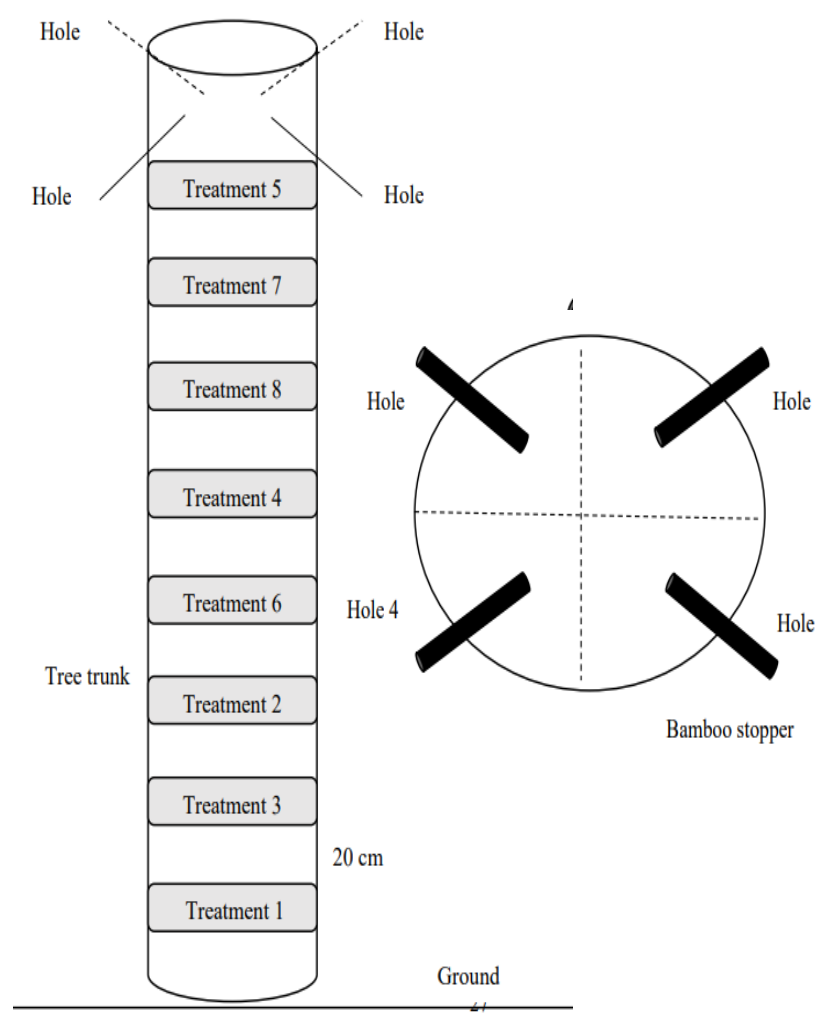

Figure 1. Illustration of experimental design of Aquilaria tree inoculation. Each tree has 8 treatments and 4 holes were made per treatment 


\section{Samples extraction for Fourier-Transform Infra Red (FTIR) analysis}

The sample extraction method was adopted from Jayachandaran et al. (2014). The harvested agarwood samples were crushed and oven-dried for 2 days at $60{ }^{\circ} \mathrm{C}$. The dried samples were extracted with sterile distilled water for 14 days. The filtrate of agarwood solution was collected and hydro-distillated using a standard Clevenger apparatus to obtain agarwood extracts. The collected extracts were dried on anhydrous sodium sulfate to remove excess water. A total of eight different agarwood extracts were produced from eight different treatments per incubation period. An FTIR analysis was performed by $\mathrm{KBr}$ Pellet Method (FTIR Alpha T Bruker, Germany) to detect the presence of agarwood chemical compounds. About $0.002 \mathrm{~g}$ of each extract was first grounded with $0.2 \mathrm{~g}$ of potassium bromide and dried for 2 hours at $120{ }^{\circ} \mathrm{C}$. The agarwood extracts were pressed to form a pellet and analyzed in IR region of $400-4000 \mathrm{~cm}^{-1}$ at room temperature (Khademolhosseini et al. 2019).

\section{Isolation and identification of fungi}

Fungal strains were collected from a naturally infected, medium-sized agarwood tree found in the same study site. Small pieces of agarwood chips $(2-5 \mathrm{~cm}$ thick) were stripped off from the region of infected Aquilaria tree bark. In the laboratory, agarwood pieces were surface-sterilized with ethanol $(96 \%)$ for 10 seconds followed by quick immersion into sodium hypochlorite $(1 \%)$ for 45 to 60 seconds. Agarwood pieces were then washed with sterile distilled water for 10 seconds and dried. Agarwood pieces were inoculated aseptically on PDA media and incubated at $28 \pm 1{ }^{\circ} \mathrm{C}$ for $2-3$ days. After incubation, fungal colonies grown on PDA media were sub-cultured to obtain a single fungal colony for DNA identification. Pure fungal isolates were cultured in SDB before extracting the genomic DNA. Fungal genomic DNA was then extracted using Zymo Research Quick-DNA Fungal/Bacterial Miniprep kit following the manufacturer's instruction. The fungal identification was done by DNA barcoding using rRNAITS sequence $(\sim 700 \mathrm{bp})$. The DNA sequence results were entered in the GenBank database with the accession numbers (Table 6). The DNA sequences were matched with the data sequences in NCBI Nucleotide Collection Database using BLAST and the top BLAST matches were selected. Phylogenetic tree of fungi was constructed using MEGA7 software (Kumar et al. 2016) using the neighborjoining method and Kimura 2-parameter was selected to calculate the evolutionary distance (Taha et al. 2021).

\section{Statistical analysis}

The total length and area of discoloration zones were determined and the mean values per treatment and per tree were calculated. All statistical analysis were performed using R 4.0.3 software (R Core Team 2020). All data were checked for normality of residuals and homogeneity of variance using the Shapiro-Wilk test and Levene's test, respectively. Wherever necessary, the data were transformed using $\log _{10}$. Data were subjected to a two-way analysis of variance (ANOVA), followed by a pairwise comparison using TukeyHSD test, with incubation period and induction treatments as factors.

\section{RESULTS AND DISCUSSION}

\section{Measurement of discoloration zones}

All induction treatments were capable of promoting agarwood formation as early as 1 month post-inoculation. After every incubation period, the outer layer of the tree bark was removed to observe the discoloration zone formed around the inoculated site. On visual inspection, dark irregular-shaped patches were observed around the inoculated region (Table 1 and Table 2). This showed that the decayed wood bark layers resulted from the infection following the induction treatments. Discoloration zone exhibited the presence of agarwood on all trees at each incubation period. A total of 384 discoloration zones were detected from all 12 trees, with both area and total length measured. It was observed that the discoloration zones appeared generally longer vertically than horizontally as the incubation period increases, which was supported by Nobuchi and Siripatanadilok (2008).

However, only the incubation period significantly affected the area and the total length of discoloration zones $(P<0.001)$, but no effect of induction treatments, and interactions between induction treatments and incubation period $(P>0.05$, Table 3$)$ were detected. Both the area and the total length of discoloration in each of 6 and 9 months of incubation period were significantly greater than 1 and 3 months of incubation period irrespective of induction treatments, including treatments from different fungal species $(P<0.05$, Table 3 and Table 4$)$. Based on visual observation on the inoculated sites of the trees, two potential fungi (UBDFC 06 Aspergillus sp. and UBDFH 16 Cladosporium sp.) are more inclined to form greater discoloration zones (pers. obs.). However, none of these observations revealed the significant effects of induction treatments on the formation of discoloration zone. There were no significant differences in the area and total length of discoloration zones between all induction treatments, including between fungal-inoculant treatments $(P>0.05$, Table 3). It can be deduced that tree holes inoculated with UBDFH25 Trichoderma sp., UBDFH27 Exophiala sp., UBDFH16 Cladosporium sp., UBDFC07 Aspergillus sp., UBDFM02 Trichoderma sp., and UBDFM06 Penicillium sp. as well as the two control induction treatments (i.e. SDB media and no inoculants) promote agarwood formation at about similar rate, thus resulting in no variation in the area and the total length of discoloration. Cui et al. (2013) reported that there were no published reports on the successful application of fungi in agarwood production yet due to inadequate induction activity of available fungi. Hence more research is required to assess microbial strains that are capable of developing high agarwood yield through methods of artificial induction (Chhipa and Kaushik 2017). 
Table 1. Agarwood formation on Aquilaria sp. after 6 months treatment with different fungal strains.

\begin{tabular}{|c|c|c|c|}
\hline Induction treatments & $\begin{array}{l}\text { Discoloration occurred after } 6 \\
\text { months incubation }\end{array}$ & Induction treatments & $\begin{array}{l}\text { Discoloration occurred after } 6 \\
\text { months incubation }\end{array}$ \\
\hline
\end{tabular}

UBDFH 25 Trichoderma sp. (MK116452)

UBDFH 27 Exophiala sp. (MK116454)

UBDFH 16 Cladosporium sp. (MK116447)

Control treatment:

No fungal inoculants

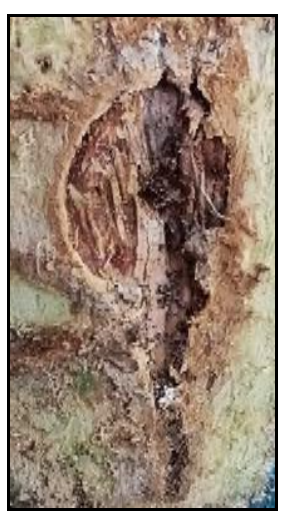

UBDFC 07 Aspergillus sp.
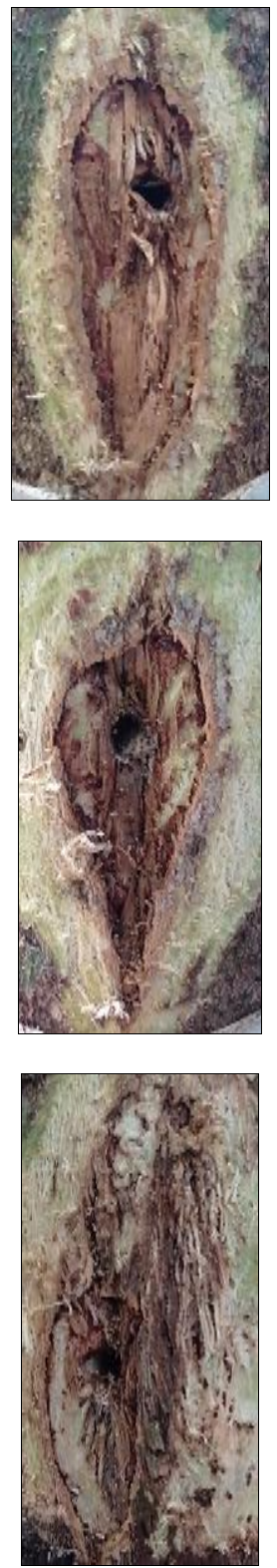
(MK116442)

UBDFM 02 Trichoderma sp. (MK116429)

UBDFM 06 Penicillium sp. (MK116432)
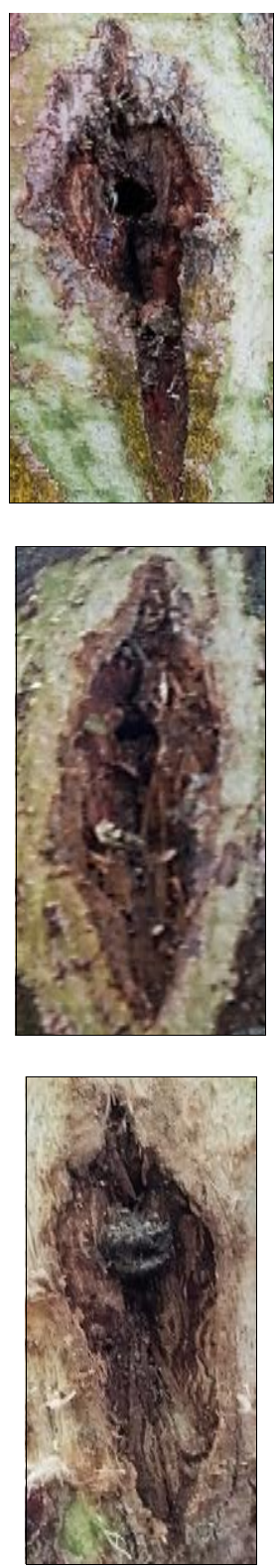

Control treatment: SDB only

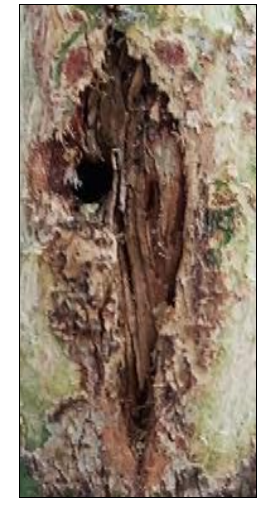


Table 2. Agarwood formation on Aquilaria sp. after 9 months treatment with different fungal strains.

\begin{tabular}{lllll}
\hline Induction treatments & $\begin{array}{l}\text { Discoloration occurred after } 9 \\
\text { months incubation }\end{array}$ & Induction treatments & $\begin{array}{l}\text { Discoloration occurred after } 9 \\
\text { months incubation }\end{array}$ \\
\hline
\end{tabular}

UBDFH 25 Trichoderma sp. (MK116452)

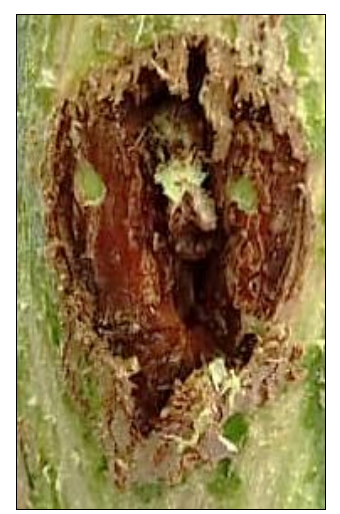

UBDFH 27 Exophiala sp. (MK116454)

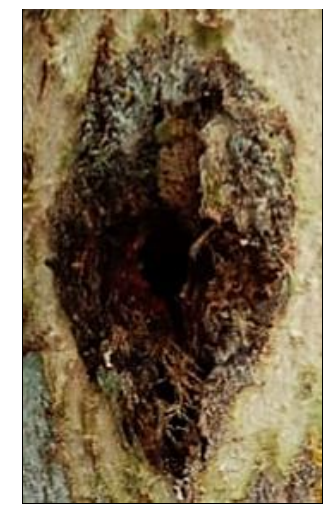

UBDFH 16 Cladosporium sp. (MK116447)

Control treatment: No fungal inoculants
UBDFC 07 Aspergillus sp. (MK116442)

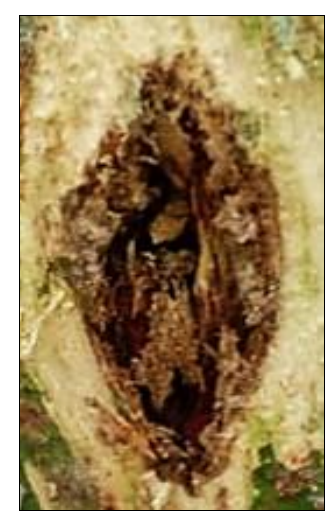

UBDFM 02 Trichoderma sp. (MK116429)

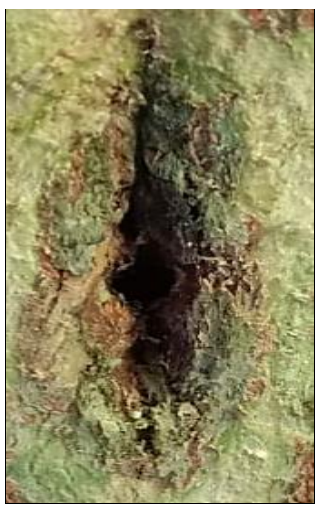

UBDFM 06 Penicillium sp. (MK116432)

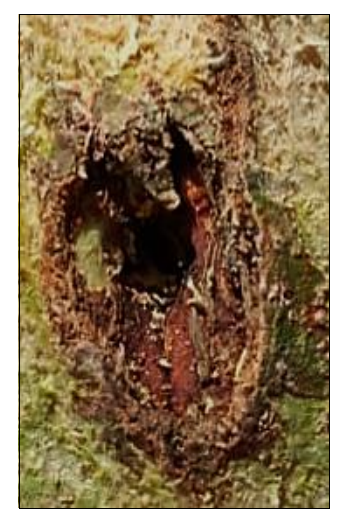

Control treatment: SDB only

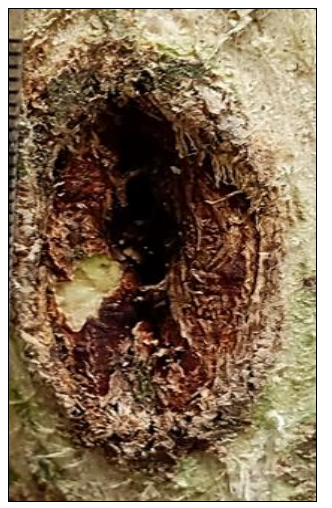


Similarly, agarwood formation was observed from the tree holes to which no inoculants has been added (Table 1 and Table 2). This notion is fully supported by Zhang et al. (2014), who observed that agarwood can also be induced by physical wounding without the presence of microbes. This finding also suggests that agarwood formation is not dependent specifically on the activity of specific fungi but rather a host typical reaction to injury or invasion (Wong et al. 2013). Agarwood formation can also be observed from the holes treated with SDB medium alone when the bark layer was removed. This finding complies with Zhang et al. (2014), who suggested the use of chemical methods as an alternative approach to induce agarwood in which wounds are deliberately treated with certain chemical reagents. Agarwood formation is the main finding of the present study that represents a part of the multifaceted alterations in a living tree that occurred after the injury and invasion by the inoculated fungi. However, the present results showed the discoloration zones formed in inoculated trees only within 9 months of incubation.

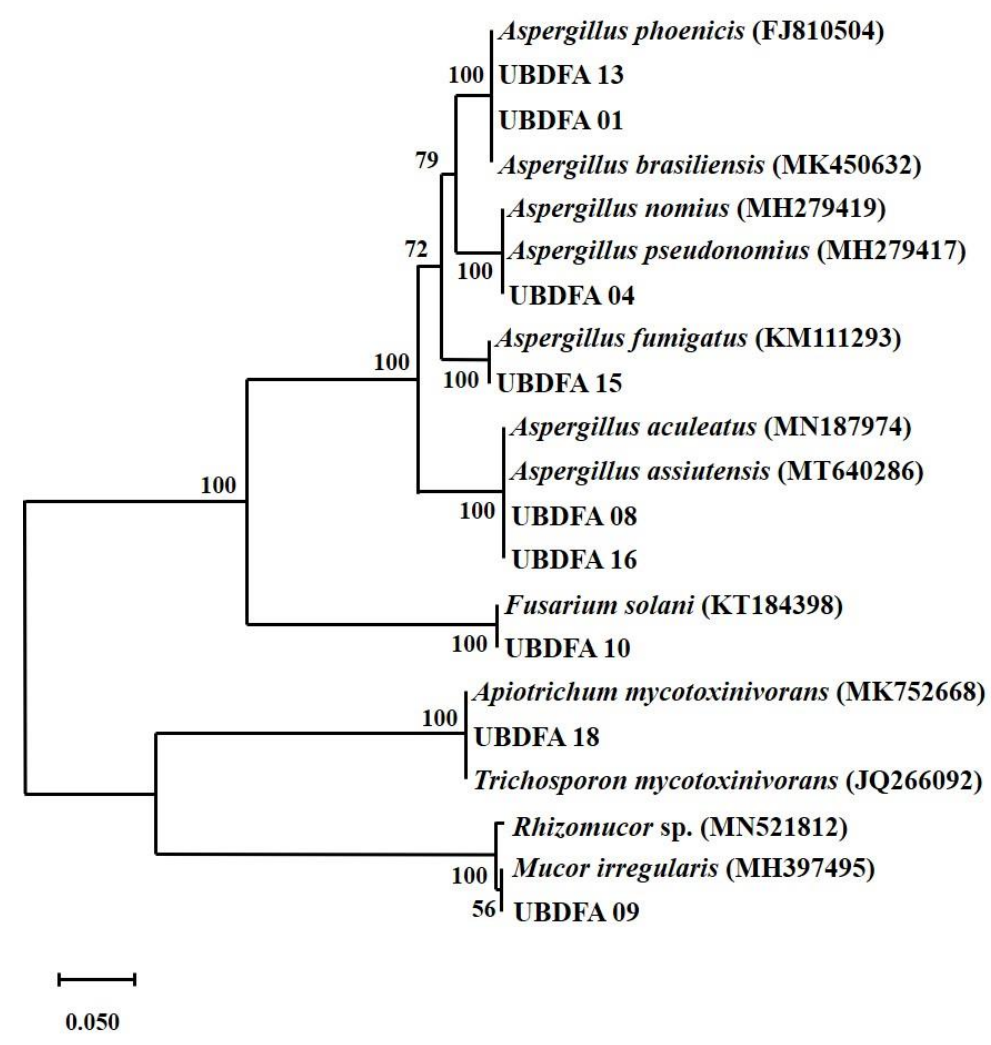

Figure 2. Phylogenetic tree of identified fungal strains based on rRNA-ITS sequence. Bootstrap percentage based on 1000 replicates were represented by the numbers at nodes. GenBank accession number is in the parenthesis. Scale represents the evolutionary distance (unit is no. of base substitutions per site)

Table 3. The effects of agarwood induction treatments and incubation periods on (a) Area of discoloration zone in $\mathrm{cm}^{2}$ and (b) Total length of discoloration zone in cm, of inoculated Aquilaria sp. Mean squares (MS), F-values and $P$ values were presented following two-way analysis of variance (ANOVA).

\begin{tabular}{|c|c|c|c|c|}
\hline \multirow{2}{*}{ Factors } & df & MS & F-value & $\boldsymbol{P}$ \\
\hline & \multicolumn{2}{|r|}{ (a) } & \multicolumn{2}{|c|}{ Area of discoloration zone $\left(\mathrm{cm}^{2}\right)$} \\
\hline Incubation period & 3 & 44.02 & 9.803 & $* * *$ \\
\hline Induction treatment & 7 & 2 & 0.445 & 0.870 \\
\hline Incubation period $\mathrm{x}$ Induction Treatment & 21 & 1.2 & 0.267 & 0.999 \\
\hline Factors & & (b) & \multicolumn{2}{|c|}{ Total length of discoloration zone $(\mathrm{cm})$} \\
\hline Incubation period & 3 & 17.335 & 10.362 & $* * *$ \\
\hline Induction treatment & 7 & 1.123 & 0.671 & 0.695 \\
\hline Incubation period $\mathrm{x}$ Induction Treatment & 21 & 0.749 & 0.448 & 0.979 \\
\hline
\end{tabular}

Note: The degrees of significance are as follow: $* P<0.05$, ** $P<0.01, * * * P<0.001$ 


\section{FTIR analysis}

The chemical properties of agarwood extracts were analyzed and the data generated were compared to references adopted from Jamahseri et al. (2014) and Syaima et al. (2016). Due to the limited amount of extracts produced from other incubation periods, only 13 agarwood extracts produced from the induction treatments of 6 months ( $n=8$ extracts) and 9 months ( $n=5$ extracts) were analyzed in this study (Table 5). The presence of $\mathrm{C}-\mathrm{H}$ bond was detected in most agarwood extracts produced from all induction treatments. The spectra in all samples showed peak position at $3000-2800 \mathrm{~cm}^{-1}$ indicating the presence of $\mathrm{C}-\mathrm{H}$ bond. The $\mathrm{C}-\mathrm{H}$ bond (aromatic alkane) has been associated with the sweet fragrance of agarwood (Nasardin et al. 2018). The presence of C - C stretching vibrations of aromatic alkanes group was also confirmed at range $1500-1400 \mathrm{~cm}^{-1}$. A broad spectrum was observed, indicating the existence of $\mathrm{O}-\mathrm{H}$ bond in the samples. The presence of $\mathrm{OH}$ group in all analyzed samples at a peak range of $3600-3400 \mathrm{~cm}^{-1}$ may indicate the presence of chemical compounds that are miscible in the distilled water such as agarospirol and jinko-eremol (Salleh 2012). However, the specific chemical compounds of agarwood could not be analyzed using FTIR.

\section{Isolation and identification of fungi}

The present study explored the diversity of fungal species exist in naturally infected agarwood. Four genera of fungal strains were isolated and identified as Aspergillus, Fusarium, Mucor, and Trichosporon (Table 6, Figure 2). Many studies have identified several genera of fungi that are associated with agarwood formation including Aspergillus and Fusarium (Subasinghe et al. 2012; Faizal et al. 2020). In this study, a different Aspergillus sp. (UBDFC07, MK116442) was used as fungal inoculants, which also resulted in discoloration (Table 1 and Table 2).

However, no prior studies have reported that Mucor and Trichosporon are associated with the formation of agarwood. This important finding has further extended the existing list of fungal genera that had previously been discovered in several agarwood studies (Subasinghe et al. 2012; Cui et al. 2013; Faizal et al. 2020). Moreover, extended studies on bacterial isolation and identification from naturally infected agarwood trees may also be conducted to produce a variety of local microbial inoculants that have the potential to induce agarwood formation in uninfected Aquilaria.

In conclusion, the present study revealed that the local species of Aquilaria beccariana and A. microcarpa have the potential to produce agarwood. In artificial induction, several fungal inoculants such as UBDFH 25 Trichoderma sp., UBDFH 16 Cladosporium sp., UBDFM 02 Trichoderma sp., UBDFH 27 Exophiala sp., UBDFH 16 Cladosporium sp., UBDFM 06 Penicillium sp., broth media (SDB) and no inoculants were applied for uninfected Aquilaria trees. Despite the insignificant difference between induction treatments, results showed that longer incubation period in all induction treatments led to greater discoloration zones in terms of area and the total length of discoloration around the inoculated site on Aquilaria tree barks. Therefore, the incubation time can be prolonged to obtain greater infection or discoloration zone, which can highly produce more agarwood. Moreover, the specific compounds of agarwood extracts could be identified using gas chromatography mass spectrometry (GC - MS). However, this study only conduct FTIR analysis to detect presence of chemical bonds associated with agarwood. This study also identified nine fungal strains from a naturally infected agarwood belonging to the genus Aspergillus, Fusarium, Mucor, and Trichosporon. Therefore, these species may need to be considered in the future manufacturing of microbial formulations to induce agarwood. This is also the first study to report the presence of Mucor and Trichosporon from a naturally infected Aquilaria tree.

Table 4. Area and total length of discolored zones on Aquilaria trees at different incubation periods.

\begin{tabular}{ccc}
\hline Incubation period & Area $\left(\mathbf{c m}^{\mathbf{2}}\right)$ & Length $(\mathbf{c m})$ \\
\hline 1-month & $2.36 \pm 0.24^{\mathbf{a}}$ & $2.27 \pm 0.16^{\mathbf{a}}$ \\
3-month & $2.47 \pm 0.14^{\mathbf{a}}$ & $2.27 \pm 0.12^{\mathbf{a}}$ \\
6-month & $4.32 \pm 0.27^{\mathbf{b}}$ & $3.99 \pm 0.22^{\mathbf{b}}$ \\
9-month & $5.07 \pm 0.28^{\mathbf{b}}$ & $3.35 \pm 0.15^{\mathbf{b}}$
\end{tabular}

Note: *Means with same letter are not significantly different at $P$ $\leq 0.05$.

Table 5. FTIR analysis of 13 agarwood extracts obtained from 6 and 9 months of incubation period

\begin{tabular}{lllc}
\hline Group Band Range (1/cm) & \multicolumn{1}{c}{ Bond } & \multicolumn{1}{c}{ Functional group } & Peak detected \\
\hline $\mathbf{3 6 0 0 - 3 4 0 0}$ & O-H & Alcohol & Detected \\
$3400-3300$ & O-H & Water & Detected \\
$\mathbf{3 0 0 0 - 2 8 0 0}$ & C-H & Aromatic alkane & - \\
$1710-1665$ & C=O stretching & A, $\beta$-unsaturated aldehydes, ketones & - \\
$1640-1630$ & H-O-H & Water & Detected \\
$\mathbf{1 5 0 0 - 1 4 0 0}$ & C-C stretching & Aromatic alkane & - \\
$1380-1350$ & C-H bending vibration -OCOCH & Alkane & - \\
$1275-1180$ & C-O stretching & Ether & - \\
$900-690$ & C-H & Aromatic ring & \\
\hline
\end{tabular}

Note: $*$ The rows in bold indicated the presence of the functional groups in the agarwood extracts. 
Table 6. Fungal identification using DNA barcoding

\begin{tabular}{ccclc}
\hline Strain ID & Identification & GenBank Accession & \multicolumn{1}{c}{ Top BLAST match } & Identity (\%) \\
\hline UBDFA 01 & Aspergillus sp. & MW876214 & Aspergillus brasiliensis & 100 \\
UBDFA 04 & Aspergillus sp. & MW876215 & Aspergillus nomius \& Aspergillus pseudonomius & 100 \\
UBDFA 08 & Aspergillus sp. & MW876216 & Aspergillus aculeatus & 100 \\
UBDFA 09 & Mucor sp. & MW876217 & Mucor irregularis & 99 \\
UBDFA 10 & Fusarium sp. & MW876221 & Fusarium solani & 99 \\
UBDFA 13 & Aspergillus sp. & MW876218 & Aspergillus brasiliensis & 100 \\
UBDFA 15 & Aspergillus sp. & MW876219 & Aspergillus fumigatus & 100 \\
UBDFA 16 & Aspergillus sp. & MW876220 & Aspergillus assiutensis \& Aspergillus aculeatus & 100 \\
UBDFA 18 & Trichosporon sp. & MW876222 & Trichosporon mycotoxinivorans & 100 \\
\hline
\end{tabular}

\section{ACKNOWLEDGEMENTS}

This study was funded by Universiti Brunei Darussalam under a research grant no. UBD/RSCH/URC/NIG/1.0/2019/003 and UBD/RSCH/1.13/FICBF(b)/2019/012. The authors thank the Forestry Department, Ministry of Primary Resources and Tourism of Brunei Darussalam for the provision of Aquilaria tree in Benutan Forest Reserves, Brunei, the Tropical Biodiversity Centre for the facilities provided and the Brunei National Herbarium (BRUN) for the identification of Aquilaria species.

\section{REFERENCES}

Chhipa H, Kaushik N. 2017. Fungal and bacterial diversity isolated from Aquilaria malaccensis tree and soil, induces agarospirol formation within 3 months after artificial infection. Front Microbiol 8: 1286. DOI: $10.3389 /$ fmicb.2017.01286

Cui J, Wang C, Guo S, Yang L, Xiao P, Wang M. 2013. Evaluation of fungus-induced agilawood from Aquilaria sinensis in China. Symbiosis 60 (1): 37-44. DOI: 10.1007/s13199-013-0237-z

Cui J, Guo S, Fu S, Xiao P, Wang M. 2013. Effects of inoculating fungi on agilawood formation in Aquilaria sinensis. Chi Sci Bull 58 (26): 3280-3287. DOI: 10.1007/s11434-013-5856-5

Faizal A, Esyanti RR, Aulianisa EN, Iriawati, Santoso E, Turjaman M. 2017. Formation of agarwood from Aquilaria malaccensis in response to inoculation of local strains of Fusarium solani. Trees (Berl.West) 31 (1): 189-197. DOI: 10.1007/s00468-016-1471-9

Faizal A, Azar AWP, Turjaman M, Esyanti RR. 2020. Fusarium solani induces the formation of agarwood in Gyrinops versteegii (Gilg.) Domke branches. Symbiosis 81 (1): 15-23. DOI: 10.1007/s13199020-00677-w

Han T, Win PP. 2016. Influence of inoculation techniques on agarwood chips formation in Aquilaria malaccensis. Int J Curr Res Life Sci 5 (7): 604-607

IUCN. 2020. The IUCN Red List of Threatened Species. Version 2021-1. www.iucnredlist.org. Downloaded on 15 March 2021.

Jamahseri NF, Rodhi MN, Zulkarnain NH, Husain NC, Masruddin AF 2014. FTIR analysis of phenolic compound as pancreatic lipase inhibitor from inoculated Aquilaria malaccensis. Malaysian J Anal Sci 18 (3): 683-689.

Jayachandaran K, Sekar I, Parthiban KT, Damodarasamy A, Suresh KK. 2014. Analysis of different grades of agarwood (Aquilaria malaccensis Lamk.) oil through GC-MS. Indian J Nat Prod Resour 5. $44-47$.

Khademolhosseini R, Jafari A, Mousavi SM, Hajfarajollah H, Noghabi KA, Manteghian M. 2019. Physicochemical characterization and optimization of glycolipid biosurfactant production by a native strain of Pseudomonas aeruginosa HAK01 and its performance evaluation for the MEOR process. RSC Adv 9 (14): 7932-7947. DOI: 10.1039/C8RA10087J

Kumar S, Stecher G, Tamura K. 2016. MEGA7: molecular evolutionary genetics analysis version 7.0 for bigger datasets. Mol Biol Evol 33 (7): 1870-1874. DOI:10.1093/molbev/mswo54
Lee SY, Mohamed R. 2016. The origin and domestication of Aquilaria, an important agarwood-producing genus. Agarwood: 1-20. DOI: 10.1007/978-981-10-0833-7_1

Nasardin NRM, Hanafiah MAM, Zainon M, Ibrahim M, Rahman AIA, Baharudin ZA, Husin MHM, Mahir I, Zulkefle AA. 2018. Comparison of chemical compounds of essential oils from natural agarwood and inoculated agarwood (Roselle-based inoculation). Indones J Electr Eng Comput Sci 11 (2): 677-681. DOI: 10.11591/ijeecs.v11.i2.pp677-681

Naziz PS, Das R, Sen S. 2019. The scent of stress: Evidence from the unique fragrance of agarwood. Front Plant Sci 10. DOI: 10.3389/fpls.2019.00840

Nobuchi T, Siripatanadilok SA. 2008. The formation of wood in tropical forest trees. In: Nobuchi T, Mohd Hamami S (eds) Cytological observations of Aquilaria crassna wood associated with the formation of aloeswood. UPM Press, Malaysia.

$\mathrm{R}$ Core Team. 2020. A language and environment for statistical computing. R Foundation for Statistical Computing, Vienna, Austria. www.R-project.org/

Rueden CT, Schindelin J, Hiner MC, DeZonia BE, Walter AE, Arena ET, Eliceiri KW. 2017. ImageJ2: ImageJ for the next generation of scientific image data. BMC Bioinformatics 18 (1): 1-26. DOI: 10.1186/s12859-017-1934-Z

Salleh N. 2012. Extraction Performance Study of Gaharu Using Microwave Extraction Method (Doctoral dissertation, UMP).

Subasinghe SMCUP, Hettiarachchi DS, Rathnamalala E. 2012. Agarwood-type resin from Gyrinops walla Gaertn: A new discovery. J Trop For Sci 2 (2). DOI: 10.31357/jtfe.v2i2.594

Syaima MTS, Adam F, Nizam ASM, Farah MZY, Saufi SMS. 2016. Molecular nano-sieve approach by the application of polypiperazine (PPA) based membrane for the recovery of water soluble agarwood (Aquilaria malaccensis) marker molecules. ARPN J Eng Appl Sci 11 (4): 2174-2178.

Taha H, Shivanand P, Shahminan NIN, Osman M, Abdul-Halim AMAA, Abdullah M. 2020. Isolation and identification of culturable bacteria and fungi from mixed dipterocarp and mangrove forests of Brunei Darussalam. Proc Natl Acad Sci India Section B Biol Sci 90 (3): 523530. DOI: $10.1007 / \mathrm{s} 40011-019-01119-4$

Taha H, Shivanand P, Zainudin MAA, Hadanan NA. 2021. Short communication: Identification of culturable marine fungi and bacteria from coastal region in Brunei Darussalam. Biodiversitas 22 (3): 13261331. DOI: $10.13057 /$ biodiv/d220332

Tian JJ, Gao XX, Zhang WM, Wang L, Qu LH. 2013. Molecular identification of endophytic fungi from Aquilaria sinensis artificial agarwood induced by pin-holes. Afr J Biotechnol 12: 3115-331.

Wong MT, Siah CH, Faridah QZ, Mohamed R. 2013. Characterization of wound responsive genes in Aquilaria malaccensis. J Plant Biochem Biot 22 (2): 168-175. DOI: 10.1007/s13562-012-0144-Z

Xu Y, Zhang Z, Wang M, Wei J, Chen H, Gao Z, Sui C, Luo H, Zhang X, Yang Y, Meng H, Li W. 2013. Identification of genes related to agarwood formation: Transcriptome analysis of healthy and wounded tissues of Aquilaria sinensis. BMC Genomics 14 (1): DOI: 10.1186/1471-2164-14-227

Zhang Z, Wei J, Han X, Liang L, Yang Y, Meng H. 2014. The sesquiterpene biosynthesis and vessel-occlusion formation in stems of Aquilaria sinensis (Lour.) Gilg Trees induced by wounding treatments without variation of microbial communities. Int J Mol Sci 15 (12): 23589-23603. DOI: 10.3390/ijms151223589 